\title{
Endoscopic dilation of benign post-esophagectomy anastomotic strictures: long-term outcomes and risk of recurrence
}

\author{
Mead Badira, Alain Suissab ${ }^{b}$ Michael Orlovskyc, Yousef Abu Asbeh', lyad Khamaysia,b \\ Technion-Israel Institute of Technology; Rambam Health Care Campus, Haifa, Israel
}

\begin{abstract}
Background Benign esophageal anastomotic strictures often require repeat dilation to relieve dysphagia. Little is known about the factors that affect their remediation. The purpose of this article was to retrospectively evaluate the long-term clinical results of endoscopic dilation in the treatment of benign anastomotic strictures after esophagectomy, and to identify factors associated with stricture recurrence.
\end{abstract}

Methods A single-center retrospective analysis (using electronic records) was performed on patients who underwent endoscopic dilation for esophageal anastomotic strictures. Long-term clinical effectiveness, including technical and clinical success, and complication rate were assessed. Factors independently related to recurrence were evaluated.

Results Between January 2014 and December 2017, a total of 35 patients who had benign anastomotic strictures after esophagectomy underwent 182 endoscopic dilation procedures. Technical success was $100 \%$. Thirty-two patients (91\%) had initial relief of dysphagia. The clinical success, defined as resolution of dysphagia and achieving luminal patency of $13 \mathrm{~mm}$ or more, was achieved in 24 patients (69\%). Strictures recurred in $43 \%$ of patients, and refractory strictures were identified in 10/35 (29\%). Proximal anastomosis and the presence of anastomotic foreign bodies were found to be risk factors for refractory strictures. The complication rate was low (4\%) and adverse events were mild. No major complications (perforations, severe bleeding) or treatmentrelated deaths occurred in this series.

Conclusions Endoscopic dilation has a high technical and a good clinical success rate. However, anastomotic strictures are often refractory and frequently recur.

Keywords Esophagectomy, anastomotic stricture, endoscopic dilation

Ann Gastroenterol 2021; 35 (1): 1-7

\section{Introduction}

Benign anastomotic strictures that develop after surgery for esophageal tumors can be particularly difficult to manage

\footnotetext{
${ }^{a}$ The Ruth and Bruce Rappaport Faculty of Medicine, Technion - Israel Institute of Technology (Mead Badir, Iyad Khamaysi); b Department of Gastroenterology (Alain Suissa, Iyad Khamaysi); 'Department of Chest Surgery, Rambam Health Care Campus (Michael Orlovsky, Yousef Abu Asbeh), Haifa, Israel
}

Conflict of Interest: None

Correspondence to: Iyad Khamaysi, MD, Director, Endoscopy Unit, Dept. of Gastroenterology, Rambam Health care Campus, Rappaport Faculty of Medicine, Technion-Israel Institute of Technology, POB 9602, Haifa 31096, Israel, e-mail: k_iyad@rambam.health.gov.il;

k_iyad@technion.ac.il; iyad.khamaysi@gmail.com

Received 4 October 2020; accepted 18 November 2020;

published online 27 January 2021

DOI: https://doi.org/10.20524/aog.2021.0590 by non-surgical means. These benign strictures develop frequently when an esophagogastric anastomosis is performed after esophagectomy, with a mean prevalence rate of $30 \%$ (range 9-48\%) [1]. In addition, a proportion of patients with benign anastomotic strictures experience recurrent strictures requiring multiple therapeutic sessions [2]. The management of these anastomotic, complex and non-peptic strictures requires multidisciplinary strategies [3]. Typically, these strictures are amenable to mechanical endoscopic dilation using a variety of endoscopic tools (Savary-Gilliard dilators or through-thescope balloons) and carry an acceptably low complication rate [4].

Cardiovascular risk factors, such as diabetes and obesity, and prior chemoradiation are associated with anastomotic stricture development after esophagectomy. A shorter time of dysphagia onset after surgery, presence of anastomotic leak, type of transthoracic approach, intraoperative hemorrhage, poor vascularization of the gastric tube, and type of anastomosis (staples worse than hand-sewn) are factors associated with stricture recurrence [3]. On the other hand, 
studies of the clinical and endoscopic factors associated with refractory strictures are lacking. Risk-stratifying patients prone to have strictures refractory to traditional endoscopic therapies may help determine the appropriate timing and relative benefit of other strategies, such as stent insertion or surgical revision. However, the prevalence of recurrent and refractory strictures using standardized criteria is not well-characterized.

In addition, endoscopic findings that influence the clinical outcome in these patients are not well delineated. A common endoscopic finding in patients with anastomotic strictures is the presence of suture material or staples protruding into the esophageal lumen. The presence of a foreign body may contribute to inflammation and scarring, thereby influencing stricture formation [5].

Therefore, the aim of our retrospective study was to evaluate the long-term clinical results of endoscopic dilation for benign anastomotic strictures after esophagectomy, to determine the rates of recurrent and refractory strictures, and to identify factors associated with recurrence of strictures.

\section{Patients and methods}

\section{Patient population}

A retrospective analysis (using electronic records) was performed on patients who underwent endoscopic dilation for esophageal stenosis in our unit (Advance Endoscopy Unit, Gastroenterology Department, Rambam healthcare campus).

The study was approved by our institutional review board (0096-17-RMB) and informed consent was obtained from each patient.

The study population included patients who developed dysphagia after formation of an esophageal anastomosis. Only patients who had an anastomotic esophageal stricture and clinical dysphagia were included.

\section{Dilation procedures}

All patients underwent fluoroscopic Savary-Gilliard mechanical dilation. During upper endoscopy, a spring-tip Savary-Gilliard metallic guidewire (Wilson-Cook Medical Inc., Winston-Salem, NC, USA) was inserted across the stenotic anastomosis, and then the dilators (polyvinyl hollow core bougie, Wilson-Cook Medical Inc., Winston-Salem, NC, USA) were inserted over the guidewire under fluoroscopic control (Fig. 1). Retention time (duration of expansion) for each dilator was about $1 \mathrm{~min}$ (variable, at the endoscopist's discretion).

Patients underwent serial dilations until successful stricture remediation was achieved. Re-intervention was performed if they developed recurrent dysphagia. Usually, procedures were performed 2-4 weeks apart with the use of 3 dilators (with $1 \mathrm{~mm}$ increment) in each procedure. In accordance with our institutional preferences, all the dilations were performed using Savary-Gilliard dilators and not with balloons.

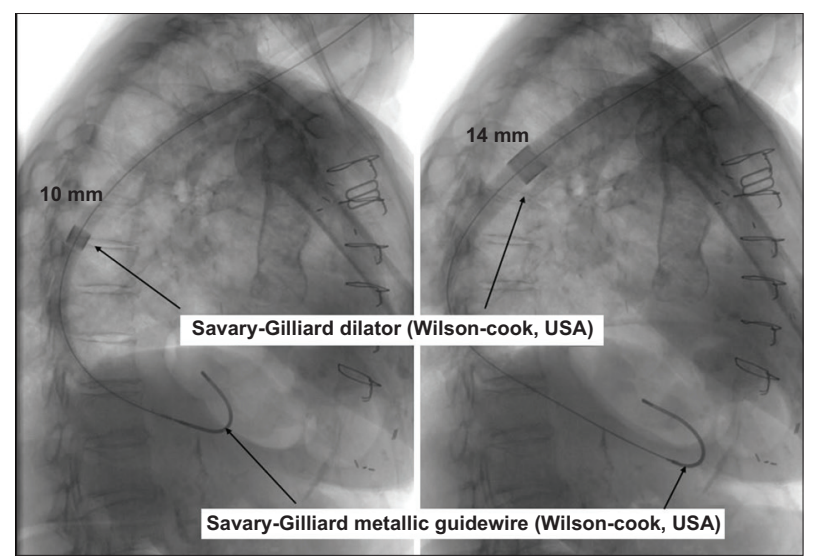

Figure 1 Fluoroscopy showing Savary-Gilliard dilation procedure

\section{Stricture characteristics}

Size (diameter) of stenosis was subjectively estimated by the operator. The locations of the anastomosis and the presence of foreign bodies were recorded. Because of its limited documented effectiveness, endoscopic steroid injection was not used. The stricture was considered proximal when the distance from the incisors was less than $20 \mathrm{~cm}$ (McKeown operation) and distal when the distance was more than $20 \mathrm{~cm}$ (Ivor-Lewis operation).

\section{Definitions of variables}

Technical success was defined as the ability to traverse the stricture with the chosen dilator and subsequent completion of dilation (increasing luminal diameter by at least $3 \mathrm{~mm}$ ).

Dysphagia scores were retrieved from the patients' medical records according to Mellow and Pinkas' dysphagia score [8]. The score consists of levels $0-4$, dependent on the type of food the patient is able to swallow, where: $0=$ no dysphagia; 1 = difficulty with solid foods; 2 = semisolid diet only; 3 = liquids only; and $4=$ total dysphagia to all foods, liquids and saliva.

Clinical improvement was defined as an improvement in dysphagia score from the baseline by at least one level. Clinical success was defined as resolution of dysphagia and achieving luminal patency ( $13 \mathrm{~mm}$ or more) for $\geq 1$ month.

A stricture was considered recurrent if, after a luminal patency of $13 \mathrm{~mm}$ was achieved, the stricture was found to be $<13 \mathrm{~mm}$ on repeat endoscopic evaluation for dysphagia within 4 weeks or less. A stricture was considered refractory if luminal patency of $\geq 13 \mathrm{~mm}$ could not be achieved after more than 10 dilation sessions in total.

\section{Statistical analysis}

Reviews of electronic medical charts, which included office visits, radiologic studies, and operative and endoscopic reports, were performed to identify baseline patient and stricture characteristics for analysis. Potential risk factors for refractory strictures were assessed by univariate and 
multivariate analyses, with the chi-square statistic in the case of categorical variables and simple logistic regression in the case of continuous variables. Risk factors included in the analysis were: type of anastomosis; time to first dilation session and numbers of sessions; existence of foreign bodies; history of anastomotic leak; and prior chemoradiation therapy.

\section{Results}

\section{Patient characteristics}

Between January 2014 and December 2017, we performed 644 fluoroscopically guided endoscopic (upper and lower) dilation procedures in 95 patients. Thirty-five patients who had benign anastomotic strictures after esophagectomy underwent a total of 182 procedures.

The characteristics of the patient population, including relevant medical history, are summarized in Table 1. They consisted of 23 men and 12 women, and the age range was 5190 years (median 63 years).

All patients underwent esophagectomy for malignant esophageal neoplasms: adenocarcinoma $n=24$ (69\%), and squamous cell carcinoma $n=11$ (31\%). These patients (35) underwent either Ivor-Lewis $(n=27,77 \%)$ or McKeown $(n=8$, 23\%) esophagectomy.

All patients suffered from moderate-to-severe dysphagia before the dilations. The average interval between

Table 1 Patients' characteristics

\begin{tabular}{|c|c|}
\hline Characteristic & Value \\
\hline Total number of patients & 35 \\
\hline \multicolumn{2}{|l|}{ Sex no. $(\%)$} \\
\hline Male & $23(65.7 \%)$ \\
\hline Female & $12(34.3 \%)$ \\
\hline Age (years), median (range) & $63(51-90)$ \\
\hline \multicolumn{2}{|l|}{ Type of esophagectomy } \\
\hline Ivor-Lewis & $27(77 \%)$ \\
\hline McKeown & $8(23 \%)$ \\
\hline History of anastomotic leak & $2(5.7 \%)$ \\
\hline \multicolumn{2}{|l|}{ Indication for esophagectomy } \\
\hline Malignant neoplasm of the esophagus & $35(100 \%)$ \\
\hline Adenocarcinoma & $24(69 \%)$ \\
\hline Squamous cell carcinoma & $11(31 \%)$ \\
\hline \multicolumn{2}{|l|}{ Medical history } \\
\hline Cardiovascular disease & $5(14.2 \%)$ \\
\hline Diabetes & $8(22.8 \%)$ \\
\hline Tobacco smoking & $22(62.8 \%)$ \\
\hline Hypertension & $20(57.1 \%)$ \\
\hline Prior chemoradiation therapy & $4(11.4 \%)$ \\
\hline
\end{tabular}

esophagectomy and the first session of dilation was 23 weeks (range 3-118 weeks). A total of 182 sessions of mechanical dilation were performed, with an average of 5.2 sessions per patient (range 1-16 sessions). Other parameters related to the anastomosis and dilation procedures are presented in Table 2 .

The follow-up period after the first dilation was up to 52 months (median 10 months). At the time this article was prepared, 30 patients were being followed, 4 patients had died of metastatic disease, and 1 patient had been lost to follow up.

\section{Clinical outcome, recurrence, and refractory strictures}

Mechanical dilation procedures were technically successful in all patients (100\%). Clinical improvement (decrease of at least 1 point in the dysphagia score) was reported by the majority of the patients after each procedure. Clinical improvement after the first session was $91 \%(32 / 35)$. Three patients with mild dysphagia (score $=1$ ) did not report clinical improvement after the first session.

Clinical success was achieved in 20 patients (57\%) with an average of 2.8 dilation procedures per patient. The rest of the patients reported a recurrence of the dysphagia after a good clinical and endoscopic results. Stricture recurrence was seen in 15 patients (43\%). In these patients, who had initially achieved luminal patency of $13 \mathrm{~mm}$ or more (Table 3), the stricture was found to be $<13 \mathrm{~mm}$ on repeat endoscopic evaluation (within 1 month) for dysphagia. These patients underwent further sessions for symptomatic relief. Clinical success (luminal patency of $13 \mathrm{~mm}$ at least) was achieved in 4 more patients (with an average of 3.5 procedures per patient within a period longer than one month). Final clinical success was achieved in 24 patients (rate $69 \%$ ).

Table 2 Dilation procedures

\begin{tabular}{lc}
\hline Characteristic & Value \\
\hline $\begin{array}{l}\text { Time period to first dilation } \\
\text { (mean } \pm \text { SD), range }\end{array}$ & $23 \pm 137,3-118$ \\
$\quad$ Median 13 weeks & \\
Stricture distance from incisors, cm & \\
$\quad \geq 20 \mathrm{~cm}$ & $27(77 \%)$ \\
$<20 \mathrm{~cm}$ (McKeown op.) & $8(23 \%)$ \\
Foreign body in the anastomosis ${ }^{* *}$ & $19 / 35(54 \%)$ \\
$\begin{array}{l}\text { Gastric food residue (during the first } \\
\text { endoscopy) }\end{array}$ & $12 / 35(34 \%)$ \\
\hline $\begin{array}{l}\text { Number of dilations (mean } \pm \text { SD), range } \\
\text { Maximal dilator size reached (mm) }\end{array}$ & $5.2 \pm 4.13,1-16$ \\
$\quad \leq 13$ mm & $8(22.8 \%)$ \\
$\quad 14-16 \mathrm{~mm}$ & $20(55.5 \%)$ \\
$\quad \geq 17$ mm & $7(20 \%)$ \\
\hline${ }^{*}$ All patients underwent fluoroscopic Savary-Gilliard mechanical dilation \\
${ }^{*}$ Suture material and staples \\
$S D$, standard deviation
\end{tabular}


Table 3 Clinical outcome of fluoroscopically guided Savary-Gilliard dilation in 35 patients with benign anastomotic strictures after esophagectomy

\begin{tabular}{|c|c|}
\hline Characteristic & Value \\
\hline Technical success $^{\star}$ & $100 \%$ \\
\hline Clinical success after the first procedure ${ }^{* *}$ & $(32 / 35) 91 \%$ \\
\hline $\begin{array}{l}\text { Dysphagia score }{ }^{* * *} \text { prior to first dilation }(0-4) \text {, } \\
\text { mean } \pm \text { SD }\end{array}$ & $2.97 \pm 1.07$ \\
\hline \multicolumn{2}{|l|}{ Median 3, range 1-4 } \\
\hline $\begin{array}{l}\text { Dysphagia score }{ }^{* * *} \text { after the first dilation }(0-4) \text {, } \\
\text { mean } \pm \text { SD }\end{array}$ & $1.62 \pm 1.2$ \\
\hline \multicolumn{2}{|l|}{ Median 2, range $0-3$} \\
\hline $\begin{array}{l}\text { Dilations to achieve initial patency }(13 \mathrm{~mm}) \text {, } \\
\text { mean } \pm S D\end{array}$ & $3.8 \pm 2.6$ \\
\hline Patients with recurrence & $15 / 35(43 \%)$ \\
\hline Refractory strictures & $10 / 35(29 \%)$ \\
\hline Stents used & $1(3 \%)$ \\
\hline Complications $s^{* * *}$ & $5(14 \%)$ \\
\hline Major complications & $0(0 \%)$ \\
\hline \multicolumn{2}{|c|}{ *Stricture could be dilated by at least $3 \mathrm{~mm}$ during initial procedure } \\
\hline \multicolumn{2}{|l|}{${ }^{*}$ Dysphagia score improvement of at least one level } \\
\hline \multicolumn{2}{|l|}{ ***Dysphagia score (Mellow-Pinkas), reference \#8 } \\
\hline \multicolumn{2}{|c|}{$\begin{array}{l}{ }_{* * * *}^{*} \text { Three patients with transient chest pain, one patient with self-limited } \\
\text { bleeding and one with mild aspiration }\end{array}$} \\
\hline SD, standard deviation & \\
\hline
\end{tabular}

Refractory strictures were identified in 10/35 (29\%). All these patients underwent more than 10 sessions (average of 11.2 procedures per patient).

One patient who had an esophageal stent inserted in our series had a stricture that was not only refractory to endoscopic dilation, but demonstrated a high degree of ineffective expansion of the luminal diameter despite repeated dilation. This patient completed 16 sessions (up to the end of follow up) until a relatively sufficient clinical response was achieved. Notably, this patient also suffered from anastomotic leak.

\section{Risk factors for refractory strictures}

According to multivariate analysis, only 2 of the tested risk factors for refractory strictures remained significant (Table 4). Six of 8 proximal (cervical) anastomoses (distance from teeth less than $20 \mathrm{~cm}$ ) were refractory (odds ratio [OR] 2.74, 95\% confidence interval [CI] 0.89-3.39; $\mathrm{P}=0.041$ ).

All the patients with refractory strictures were found to have a foreign body in the anastomosis (suture material and/ or staples). Anastomotic foreign bodies increased the risk for refractory strictures by 3.74 ( $95 \% \mathrm{CI} 1.88-4.38 ; \mathrm{P}=0.044)$.

All other parameters (type and location of anastomosis, time to first dilation session and number of sessions, history of anastomotic leak, and prior chemoradiation therapy) were not found to be significant predictors associated with refractory strictures (Table 4).

\section{Adverse events}

Three patients suffered from transient chest pain. One of these patients underwent a gastroscopy and was diagnosed with mild self-limited post-procedural bleeding. One patient suffered from mild self-limited aspiration from gastric food residue. The overall complication rate was $14 \%$. No major complications (perforations, severe bleeding) or treatmentrelated deaths occurred in this series.

\section{Discussion}

Esophageal stenosis is a frequently encountered problem. Anastomotic strictures are one of the common benign causes among others such as peptic, caustic or radiation injury, Schatzki's rings, and esophageal webs [4-6]. Through the years, dilation has been the customary treatment for esophageal strictures. However, dilation techniques have evolved, from the use of whalebones and rigid bougies in the 17th and 18th centuries, to the widely used wire-guided, fluoroscopically controlled, flexible, polyvinyl bougies (Savary- Gilliard), and through-the-scope balloon dilators in the current era [5].

The prevalence of anastomotic strictures after esophagectomy is relatively high. These benign strictures may develop as a result of collagen deposition and fibrin production from deep ulceration or chronic inflammation [5]. The effect of medical therapy (i.e., proton pump inhibitors) can be limited in the absence of an active peptic component. Endoscopic dilation can be regarded as the primary treatment method and is associated with low complication rates [4-6].

Strictures that do not respond to standard dilation techniques pose a particular challenge. Strictures that cannot be adequately dilated for relief of dysphagia in numerous sessions, or that need multiple and frequent dilation sessions after achieving an adequate luminal diameter, may be considered refractory. There is no universally accepted definition for a refractory esophageal stricture, and definitions vary in different series [7].

In this study we included 35 patients with symptomatic post-esophagectomy anastomotic stricture (Table 1). Most of the patients underwent an Ivor-Lewis procedure with distal anastomosis. Relatively very few patients had neoadjuvant chemoradiotherapy and only 2 patients had a post-surgical anastomotic leak (Tables 1,2).

According to our institutional practice guidelines, and in order to limit potential adverse events, all strictures in this series were dilated at least 3 weeks (range 3-118 weeks) after the formation of the anastomosis. The intervals between the sessions were highly variable (range 1-12 weeks) according to the center's availability and patients' needs and compliance.

All patients underwent fluoroscopically controlled SavaryGilliard mechanical dilation. Prior studies have shown little difference in outcomes when comparing balloon dilators (exerting only radial force) to Savary-Gilliard dilators (exerting both radial and axial forces) [10]. 
Table 4 Risk factors for refractory stricture

\begin{tabular}{lcccc}
\hline Risk factors & Refractory (10) & Non-Refractory (25) & Multivariate analysis & P-value \\
\cline { 3 - 4 } & & & OR $(95 \% \mathrm{CI})$ \\
\hline Sessions number (average) & 11.2 & 2.8 & $2.30(1.40-3.71)$ & $2.74(0.89-3.39)$ \\
\hline Proximal anastomosis & 6 & 2 & $3.74(1.88-4.38)$ \\
Distal anastomosis & 4 & 23 & 0.041 \\
Foreign body in the anastomosis & 10 & 9 & $\mathrm{n} / \mathrm{s}$ \\
Time to first dilation session (mean, weeks) & 25 & 21 & $\mathrm{n} / \mathrm{s}$ \\
\hline Prior chemoradiation therapy & 2 & 1 & $\mathrm{n} / \mathrm{s}$ \\
\hline History of anastomotic leak & 1 & 5 & $\mathrm{n} / \mathrm{s}$ \\
\hline Maximal dilator size reached $>13 \mathrm{~mm}$ & 3 & & \\
\hline OR, odds ratio; CI confidence interval; $n / \mathrm{s}$, non-significant & & 2 &
\end{tabular}

Technically, all the procedures were successful. Based on the change in dysphagia score, clinical improvement (decrease of at least 1 point in the dysphagia score) was reported by the majority of the patients after each procedure. Clinical improvement after the first session was seen in 91\% (32/35).

Clinical success was achieved within 1 month in 20 patients, with an average of 2.8 dilation procedures per patient (Table 3 ). Another 4 patients achieved clinical success within a period of more than 1 month, with an average of 3.5 procedures per patient. The overall clinical success rate was $69 \%$ (24 patients).

Dilation requires a commitment to compliance by both the patient and the endoscopist, as evidenced by the large number of dilation sessions in our cohort (5.2 per patient) over an extended period of time (3-118 weeks) to achieve initial patency. Furthermore, while the majority of patients achieved initial patency and symptomatic relief, many had stenosis recurrence (up to $43 \%$ ). This high recurrence rate is similar to other studies [11]. Moreover, there was a high rate of refractory strictures (29\%) in our cohort, based on previously mentioned criteria. Recurrent and refractory anastomotic strictures may be the consequence of scar-induced luminal compromise or fibrosis without evidence of inflammation on endoscopy [12]. The high prevalence of recurrence and refractory strictures supports the idea that the likely underlying mechanism for these strictures is not easily remediated with mechanical and transient radial forces.

As stated before, cardiovascular risk factors and prior chemoradiation are associated with anastomotic stricture development after esophagectomy. Shorter time of dysphagia onset after surgery, presence of anastomotic leak, type of transthoracic approach, intraoperative hemorrhage, poor vascularization of the gastric tube, and type of anastomosis (staples worse than hand-sewn) are factors associated with stricture recurrence [3].

In our study, among the potential risk factors for refractory strictures that could be examined, only 2 remained significant on multivariate analysis (Table 4). Six of 8 proximal (cervical) anastomoses (distance from teeth less than $20 \mathrm{~cm}$, McKeown operation) were refractory (OR2.74, 95\%CI0.89-3.39; $\mathrm{P}=0.041$ ). In other words, a statistically significant correlation was found between the surgical technique used for esophagectomy (Ivor-Lewis or McKeown) and the development of refractory anastomotic stricture. The McKeown esophagectomy is similar to the Ivor-Lewis esophagectomy, except that the anastomosis is created in the neck through a separate cervicotomy, rather than via intrathoracic anastomosis [13]. Patients who underwent a McKeown esophagectomy were more prone to recurrences after dilation than were those who had an IvorLewis esophagectomy.

This difference in recurrence rate might be partly explained by the findings of others [13], who reported that anastomotic leak and stricture have been shown to occur with greater frequency in patients who underwent McKeown esophagectomy, rather than the Ivor-Lewis approach.

Another possible explanation for the higher recurrence rate seen in patients with McKeown esophagectomy might be related to the difference in the location where the anastomosis is created during surgery. The gastric tube is pulled up higher in the cervical esophagus to form the anastomosis during the McKeown surgery compared with the Ivor-Lewis surgery [13]. This maneuver might have contributed to poorer vascularization of the gastric tube at the anastomotic site, known to be a risk factor for developing postoperative anastomotic strictures [3].

A common endoscopic anastomotic finding in these patients is the presence of suture and/or staples protruding into the lumen. The presence of a foreign body may interfere with the healing process [5]. In our series, all the patients with refractory anastomotic strictures were found to have foreign bodies. The presence of anastomotic foreign bodies increased the risk for refractory stricture by 3.74 (95\%CI 1.884.38; $\mathrm{P}=0.044$ ). While removal of staples and sutures might be thought beneficial to accelerate time to initial patency and result in a longer dysphagia-free interval, no attempts were made to remove the foreign bodies. Larger studies will be necessary to validate the impact of this observation and potential endoscopic intervention.

The other tested parameters were not found to be significant predictors associated with refractory strictures (Table 4), or could not be reliably examined because of the small numbers of cases. One patient was diagnosed with anastomotic leak 
and empyema. The empyema was drained and the fistula was closed by endoscopic over-the-scope clip (Ovesco Endoscopy $\mathrm{GmbH}$, Tübingen, Germany). Later on, the patient developed a stiff fibrotic stricture that required more than 10 sessions (refractory). A partially-covered esophageal stent (WilsonCook Medical Inc., Winston-Salem, NC, USA) was inserted across the stricture. One month later, a massive tissue overgrowth was noticed on both proximal and distal flanges, so another fully-covered stent (Wallflex, Boston Scientific, Natick, MA, USA), was inserted coaxially into the first stent in order to ablate the tissue ingrowth and facilitate the extraction of the stents (Fig. 2). One week later, both stents were removed successfully. Unfortunately, the stenosis recurred and the patient needed to undergo further endoscopic dilation sessions.

Delayed gastric emptying is one of the major complications, occurring in $15-39 \%$ patients after esophagectomy [14]. It is widely believed that bilateral vagotomy and increased pyloric resistance are the major culprits. Patients suffering from delayed gastric emptying mainly complain of nausea, vomiting, anorexia, early satiety, bloating and abdominal pain, but without any evidence of mechanical obstruction. However, in some cases delayed gastric emptying symptoms might be interpreted as obstructive symptoms related to anastomotic stricture (especially in cases with distal anastomosis). The amount of

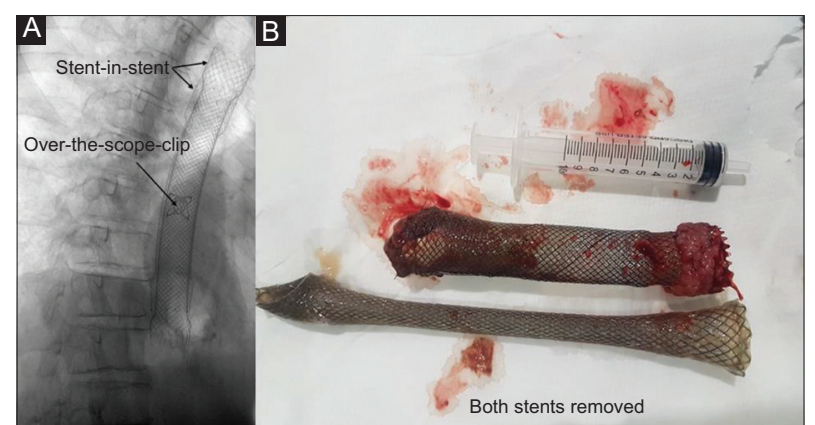

Figure 2 (A) Fluoroscopy showing over-the scope-clip and fullycovered stent inside partially-covered stent. (B) The 2 stents after removal. Note the tissue overgrowth and ingrowth on the flanges of the partially-covered stent food residue is closely related to delayed gastric emptying in esophagectomized patients [15]. In our study, and in spite of appropriate preparation, gastric food residue was found in $12 / 35(34 \%)$ of patients during the first endoscopic dilation session. Gastric food residue can substantially interfere with the quality of the endoscopic procedures and also increases the risk of aspiration. It remains controversial whether the resection type affects the postoperative gastric emptying [16]. Although the food residue was observed more often in IvorLewis anastomosis than after McKeown anastomosis, the difference was insignificant in the present study.

There were no major complications in this study (Table 3 ). Apart from resolving chest pain, mild aspiration and selflimited bleeding, our low complication rate was comparable to previous studies [9]. It is our institutional practice to delay endoscopic procedures until at least 2-3 weeks after the operation. All strictures in this series were dilated after at least 3 weeks (Table 2). This strategy might be one of the reasons for the low complication rate. All endoscopic sessions were performed with the patient in the left lateral decubitus position with reverse Trendelenburg tilting. While not yet proved, the tilted position can potentially reduce the risk of aspiration during the endoscopy.

The principal limitation of this study is its retrospective and nonrandomized design, which could have introduced some bias and decreased the study's statistical power. Besides the relatively modest number of patients, our data are based on an expert provider at a single tertiary care center, which may preclude generalizability to community practice. However, anastomotic stricture patients often have multiple comorbidities and are typically managed at large referral centers.

To summarize, fluoroscopically controlled Savary-Gilliard mechanical dilation is a well-standardized and technically easy modality for the treatment of benign anastomotic stricture after esophagectomy. The procedure is associated with an acceptably good clinical success rate and a low complication rate. However, the recurrence rate is rather high, with McKeown esophagectomy and the presence of anastomotic foreign bodies being associated with refractory strictures (Fig. 3).

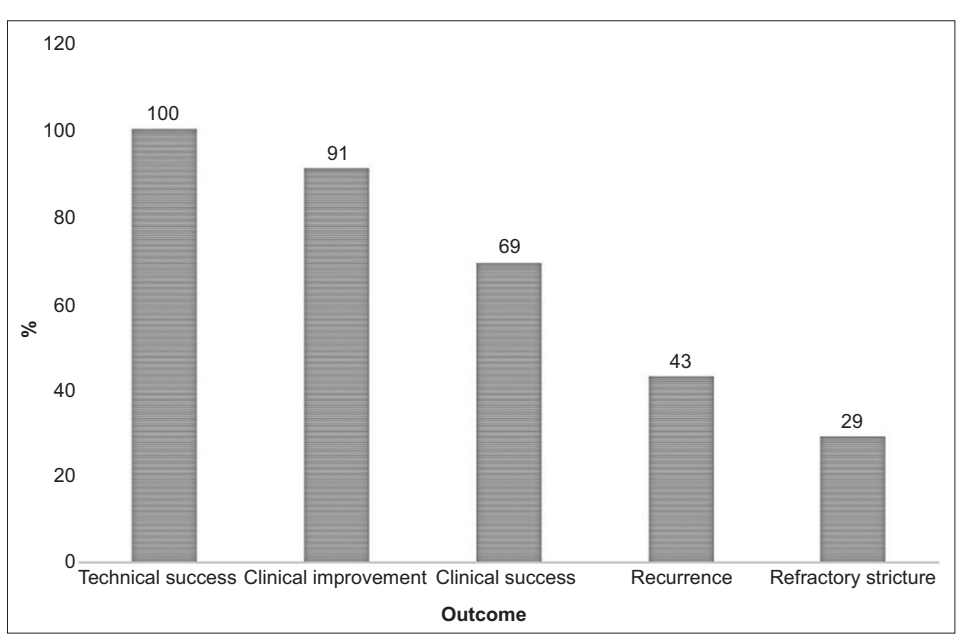

Figure 3 Chart showing the outcome of endoscopic treatment for benign esophageal anastomotic strictures 
In conclusion, this study demonstrated the following: 1) endoscopic dilation of anastomotic strictures after esophagectomy has excellent technical success and a low complication rate; 2) though the clinical success rate is good, anastomotic strictures require frequent dilation sessions and carry a high recurrence rate; and 3) many anastomotic strictures are refractory to endoscopic dilation.

\section{Summary Box}

\section{What is already known:}

- Esophageal anastomotic strictures often require repeat dilation to relieve dysphagia

- Little is known about the factors that affect their remediation

\section{What the new findings are:}

- Endoscopic dilation of anastomotic strictures after esophagectomy had an excellent technical success and a low complication rate

- Clinical success was good, but anastomotic strictures required frequent dilation sessions and carried a high recurrence rate

- Proximal anastomosis and the presence of anastomotic foreign bodies were found to be risk factors for refractory strictures

- Many anastomotic strictures were refractory to endoscopic dilation

\section{References}

1. Pierie JP, de Graaf PW, Poen H, van der Tweel I, Obertop H. Incidence and management of benign anastomotic stricture after cervical oesophagogastrostomy. Br J Surg 1993;80:471-474.

2. Honkoop P, Siersema PD, Tilanus HW, Stassen LP, Hop WC, van
Blankenstein M. Benign anastomotic strictures after transhiatal esophagectomy and cervical esophagogastrostomy: risk factors and management. J Thorac Cardiovasc Surg 1996;111:1141-1146.

3. van Heijl M, Gooszen JA, Fockens P, Busch OR, van Lanschot JJ, van Berge Henegouwen MI. Risk factors for development of benign cervical strictures after esophagectomy. Ann Surg 2010;251:1064-1069.

4. Said A, Brust DJ, Gaumnitz EA, Reichelderfer M. Predictors of early recurrence of benign esophageal strictures. Am J Gastroenterol 2003;98:1252-1256.

5. Lew RJ, Kochman ML. A review of endoscopic methods of esophageal dilation. J Clin Gastroenterol 2002;35:117-126.

6. Pereira-Lima JC, Ramires RP, Zamin I Jr, Cassal AP, Marroni CA, Mattos AA. Endoscopic dilation of benign esophageal strictures: report on 1043 procedures. Am J Gastroenterol 1999;94:1497-1501.

7. Kochman ML, McClave SA, Boyce HW. The refractory and the recurrent esophageal stricture: a definition. Gastrointest Endosc 2005;62:474-475.

8. Mellow MH, Pinkas H. Endoscopic therapy for esophageal carcinoma with Nd:YAG laser: prospective evaluation of efficacy, complications, and survival. Gastrointest Endosc 1984;30:334-339.

9. Kochman ML. Minimization of risks of esophageal dilation. Gastrointest Endosc Clin N Am 2007;17:47-58.

10. Cox JG, Winter RK, Maslin SC, et al. Balloon or bougie for dilatation of benign oesophageal stricture? An interim report of a randomised controlled trial. Gut 1988;29:1741-1747.

11. Park JY, Song HY, Kim JH, et al. Benign anastomotic strictures after esophagectomy: long-term effectiveness of balloon dilation and factors affecting recurrence in 155 patients. AJR Am J Roentgenol 2012;198:1208-1213.

12. Kim TJ, Lee KH, Kim YH, et al. Postoperative imaging of esophageal cancer: what chest radiologists need to know. Radiographics 2007;27:409-429.

13. Levy RM, Wizorek J, Shende M, Luketich JD. Laparoscopic and thoracoscopic esophagectomy. Adv Surg 2010;44:101-116.

14. Li B, Zhang JH, Wang C, et al. Delayed gastric emptying after esophagectomy for malignancy. J Laparoendosc Adv Surg Tech A 2014; 24:306-311.

15. Nakabayashi T, Mochiki E, Garcia M, et al. Gastropyloric motor activity and the effects of erythromycin given orally after esophagectomy. Am J Surg 2002;183:317-323.

16. Schmidt CE, Bestmann B, Küchler T, Schmid A, Kremer B. Quality of life associated with surgery for esophageal cancer: differences between collar and intrathoracic anastomoses. World J Surg 2004;28:355-360. 